\title{
Understanding the role of a Digital Twin in Integrated Vehicle Health Management (IVHM)*
}

\author{
Cordelia Mattuvarkuzhali Ezhilarasu, Zakwan Skaf, and Ian K Jennions
}

\begin{abstract}
Integrated Vehicle Health Management (IVHM) aims to support Condition-Based Maintenance (CBM) by monitoring, diagnosing, and prognosing the health of the host system. One of the technologies required by IVHM to carry out its objectives is the means to emulate the functioning of the host system, and the concept of a Digital Twin (DT) was introduced in aerospace IVHM to represent the functioning of such a complex system. This paper aims to discuss the role played by DT in the field of IVHM. A DT is the virtual representation of any physical product, that is used to project the functioning of the product at a given instance. The DT is used across the lifecycle of any product, and its output can be customized depending upon the area of application. The DT is currently popular in industry because of the technologies like sensors, cloud computing, Internet of Things, machine learning, and advanced software, which enabled its development. This paper discusses what encompasses a DT, the technologies that support the DT, its applications across industries, and its development in academia. This paper also talks about how a DT can combine with IVHM technology to assess the health of complex systems like an aircraft. Lastly, this paper presents various challenges faced by industry during the implementation of a DT and some of the possible opportunities for future growth.
\end{abstract}

\section{INTRODUCTION}

The recent trend of using the concept of a Digital Twin (DT) in any industry from aerospace to agriculture, from a product-oriented environment to a process or a serviceoriented environment, is due to its versatile nature and its ability to provide a solution for any given scenario. The term 'Digital Twin' was initially coined by Dr. Michael Grieves from the University of Michigan in 2002 to represent the digital equivalent of a physical asset [1]. Later, in 2012, NASA adapted DT as an integrated multi-physics, multi-scale probabilistic simulation model of a vehicle or a system that integrates best available physical models, sensor data from onboard health monitoring systems, fleet maintenance history and other such information to mirror the life of its physical twin [2]. Bolstered by the parallel growth in sensors and communication technologies, cyber-physical systems, computational speed, and artificial intelligence (AI), the term DT has been subsequently used by industry for several applications. A Gartner survey in 2019 mentions that around $75 \%$ of the industries that use Internet-of-Things (IoT) are either using DT already or plan to use it within the next year
[3]. With the given advanced technologies, the DT is currently the key to optimize the product design process, to enable smart manufacturing with Industry 4.0, to streamline the process of Maintenance Repair and Overhaul (MRO) and to reduce the overall cost involved in the product and the process life cycles.

This paper focuses on how the DT can be combined with Integrated Vehicle Health Management (IVHM) to enable Condition-Based Maintenance (CBM) of vehicles like an aircraft. $\mathrm{CBM}$ is a program that provides maintenance plans based on the condition of the vehicle [4]. It is a real-time product performance measure, which has replaced the conventional time-based programs like scheduled inspections and preventive maintenance [5]. CBM enables customized maintenance planning of the targeted components and systems of a vehicle by monitoring their health condition. This results in saving time and cost involved due to unplanned downtime, and increases the availability of the vehicle. A fully functional IVHM system assists in acquiring and analyzing health data of a vehicle to optimize the suitable maintenance plans for $\mathrm{CBM}$ [6].

Integrated Vehicle Health Management (IVHM) is defined as the technology that uses the sensor data from a vehicle to carry out diagnosis and prognosis to assess and predict the health of its systems. IVHM technology was initially introduced by NASA to collect data, diagnose, predict and mitigate faults, support operational and post-maintenance activities in space vehicles. Later, it expanded to other vehicles like aircraft and ships and evolved as a paradigm-shift to support Condition-Based Maintenance (CBM) of the vehicles. IVHM aims to ensure that the host system functions as intended, without any unexpected failure [6]. With the help of IVHM systems, the maintenance cost can be brought down significantly by predicting the system health in advance and supporting CBM.

Fig. 1 shows the general role of IVHM technology in a product's (vehicle) lifecycle [7]. In this figure, the steps involved in IVHM are explained via the Sense-AcquireTransfer-Analyze-Act (SATAA) cycle as shown in the righthand side, and its role concerning the product's lifecycle is shown in the left-hand side. To monitor the health of the system, IVHM uses state-of-the-art sensors to acquire health information from the system. It also gets input from various stages of the product's lifecycle, like design-engineering

\footnotetext{
* This work has been funded by The Boeing Company as part of their industrial collaboration with the Cranfield University IVHM Centre.

C.M. Ezhilarasu,corresponding author, (e-mail: c.m.ezhilarasu@cranfied.ac.uk),Z.Skaf(e-mail: z.skaf@cranfield.ac.uk),and I.K. Jennions (e-mail: i.jennions@cranfied.ac.uk) are with IVHM Centre, Cranfield University, United Kingdom, MK430AL.
} 
Figure 1: The role of IVHM in product lifecycle [7]

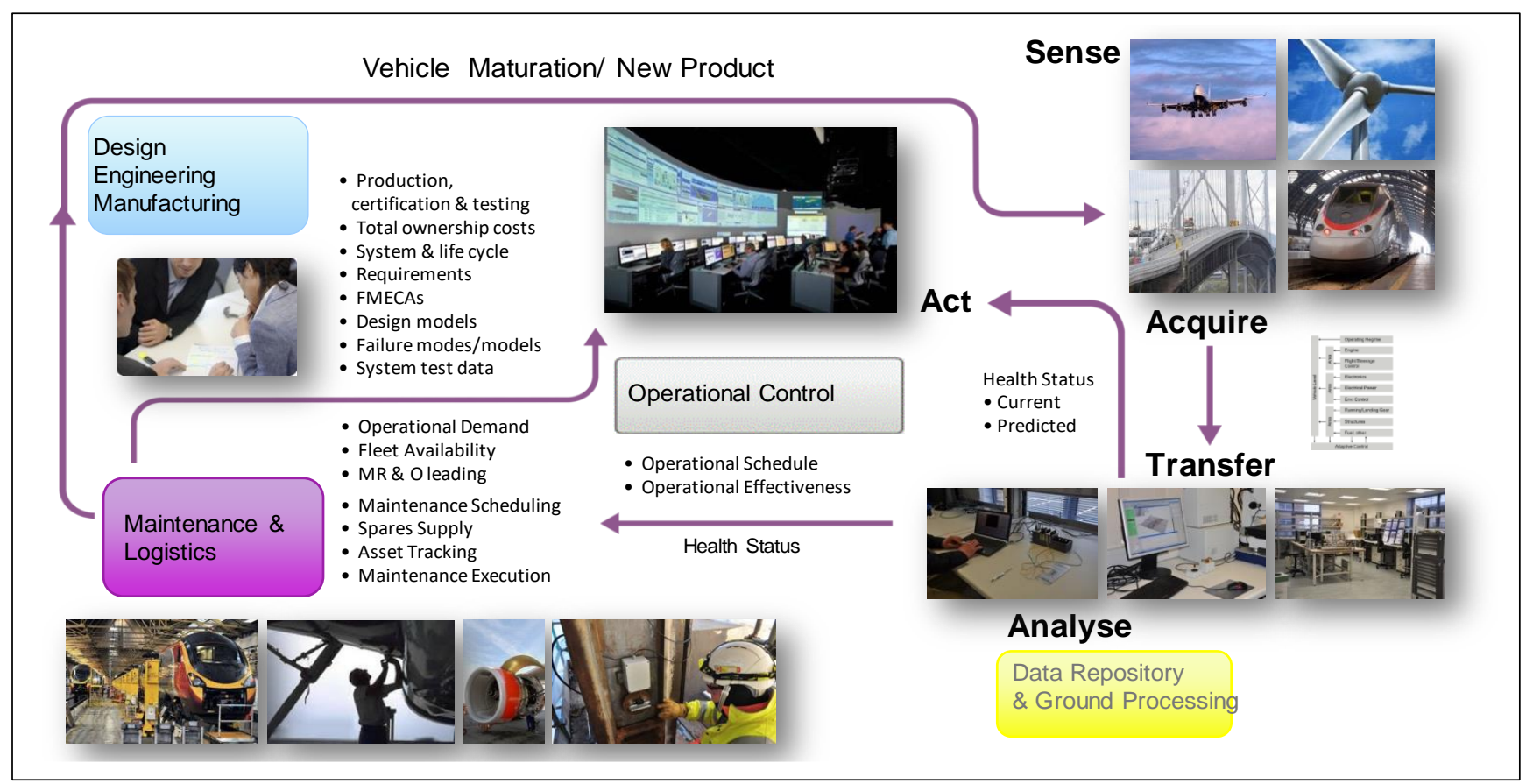

manufacturing and maintenance-logistics. Once the data is acquired, they are transferred using high-end communication technologies to analyze the health information collected from the data. The IVHM system needs high computational speed for processing all the data collected and depends upon advanced machine learning algorithms and AI for diagnosing faults and predicting the remaining useful life of the systems. This information on the system's health is provided for maintenance of the vehicle (as shown in Fig. 1), under the CBM program.

For the diagnostics and prognostics to produce effective results, the IVHM system requires a knowledge-rich representation of the host system, that could provide all possible outcomes of multiple health scenarios concerning the host system [5]. It is at this capacity that the Digital Twin (DT) comes into the picture of IVHM. The DT is a high-fidelity simulation, that can provide the virtual representation of the physical asset at a particular instant and produce what-if simulations for a variety of scenarios depending upon the data acquired [8]. This feature of DT helps in forecasting the health of the systems that could be used by IVHM. In the last five years, many aerospace and automotive industries have started using DT at various stages of a product lifecycle.

This paper explores the field of Digital Twin (DT), its design, and its different applications in IVHM. To that effect, this section introduced the fields of DT, CBM, and IVHM. Section II presents the core elements of a DT, its functions alongside the various stages of a product's lifecycle, and its enabling technologies. Section III surveys some of the applications of DT in each stage of the product's lifecycle to understand its capabilities. Section IV presents a basic IVHM system and the role of DT inscribed in it. It also talks on the developments of DT concerning the field of IVHM. It is to be noted that the majority of applications presented are from aerospace, and only a few are from of other vehicles. Section $\mathrm{V}$ discusses various challenges faced during the implementation of DT, along with gaps and opportunities in IVHM. Section VI presents the conclusion regarding the role of a DT within the field of IVHM.

\section{BUILDING BLOCKS OF A DigitAL TWIN}

At its core, the DT is defined as a simulation model representing a physical asset, but unlike the usual simulation model, the DT evolves with the life of the physical asset, factoring in age-related performance and failure. It functions as a living virtual representation of the asset. It relates to the data captured from different stages of the asset's life cycle to create the contextual environment under which the asset is operating, and its output varies based on the services expected from it. Across the literature, when a DT is referred to, it could either mean the virtual representation of a product at an instant or encompass its entire life cycle [1]. Sometimes, it also refers to the entire environment involving the physical, virtual models, data connections between them, and the nature of output [9]. Similarly, the number of building blocks of a DT also differs with the literature, ranging from three ([1], [10], [11]) to five [9], but they essentially have the same elements listed in a different order of importance.

This paper refers to the DT as the living representation of a physical product and the Digital Twin Ecosystem [12] as the environment that includes the other elements that help in the functioning of the DT. A Digital Twin Ecosystem is thus built upon the following blocks (as shown in Fig. 2): i) the product life cycle (top layer of Fig.2), ii) a virtual model or the DT (bottom layer of Fig. 2), and iii) a connecting segment (the middle layer of Fig. 2). The connecting segment is characterized by data that relates the product as a physical asset to the DT as its corresponding virtual model. The transfer of data between the top and the bottom layer of Fig. 2 via its middle layer is made possible with the help of several technologies which are listed in the right-side middle layer of Fig. 2. The following subsections discuss these central 
Figure 2: Digital Twin Ecosystem along the product lifecycle



elements of a DT in detail. It is to be noted that the MRO section of Fig. 2 is highlighted, as this paper focuses on a DT that could be used mainly for IVHM.

\section{A. Product Lifecycle}

As shown in the top layer of Fig. 2, from a product's Beginning of Life (BOL) to its End of Life (EOL), it goes through various stages during its life cycle. The product can be anything from a particular component of a system (e.g., compressor blade in an aircraft engine) or an entire system (e.g., an aircraft engine) itself. The product information passes from its BOL to the EOL, and the data generated during the lifecycle, from concept definition, design and development, and manufacturing are used as a source of the virtual model for the DT. They also serve to validate the virtual model, which can later be considered as the reference model, against which the performance and health of the product can be measured when it is in use.

\section{B. Virtual model}

The virtual representation/ DT of a product is a highly adapted simulation model (bottom layer of Fig. 2). The DT evolves alongside its physical asset based on the knowledge gained at each stage. It is the virtual representation of the physical asset at an instant and reflects on its operational behavior. It utilizes various sources ranging from geometric or 3D models, thermal or CFD models, physics-based or functional models to represent the functioning of the product [13]. It also uses data like Bill of Materials (BOM) from 'asplanned', 'as-built', and 'as-maintained' states of the product, historical information, fleet data, and maintenance records to update the contextual knowledge of the virtual model and its environment to represent the actual working condition of the product through its lifecycle (Fig. 2).

\section{The Connecting segment}

As mentioned earlier, the connecting segment (the middle layer of Fig. 2) is characterized by the data that connects physical and virtual models and by the output/ feedback from the DT. Data generated from the onboard sensors, inspections, fleet histories, and other sources during various stages of the lifecycle must be fused with the existing virtual models in order to update the DT at that stage of the product lifecycle. This data fusion within the DT ecosystem enables better model performance, adaptiveness, and robustness in the virtual models [12].

In the middle layer of Fig. 2, the data with the yellow background are input to the DT from the product and the data with the green background are the output from the DT to the product at a certain stage of its lifecycle. The output and feedback from the DT are dependent on the application. In the case of IVHM, the DT is expected to provide some of the results required to detect anomalies, health degradation, faults, and calculate the remaining useful life of the product. These outputs also change with respect to the lifecycle of the product (Fig. 2). For example, at the design stage, the DT is more useful in validating and optimizing the design process, whereas, in the manufacturing stage, the DT is used for smart factories, to visualize and streamline the process, to increase efficiency and to resolve conflicts on the shop floor [14]. The information from all stages of the product's lifecycle and the DT is sent as feedback (dotted lines in Fig. 2). This feedback information is used to generate knowledge that is required for the optimization of the product as well as the process. The connecting segment, i.e., the transfer of data between the physical and the virtual model is enabled by several advanced technologies, without which, it would be impossible to implement the DT.

\section{Enabling Technologies}

Some of the technologies that helped the successful implementation of DT (as shown in the right-side middle layer of Fig. 2) are as follows:

Sensors: The advancement in the field of sensor technologies is one of the primary enablers of any DT. Currently, there is a wide range of sensors at a lower price than in the past, and they produce abundant data, giving rise to Big Data. This data, if processed correctly, could provide 
information and the knowledge required to make the virtual model live.

Connectivity: Internet-of-Things (IoT) and cloud computing are two disruptive technologies that changed the perspective of every industry. IoT enables connectivity between various systems with an IP address, which makes it possible for data collection, and processing; it is one of the critical factors required for real-time monitoring of any process. On the other hand, cloud computing allows largescale data processing and storage in the servers, making them accessible by the internet. It makes scalable computing available at any point, which is not possible through the local servers. Edge computing carries out computation at the end of the local network before updating to the cloud, thus reducing the burden on the server [15]. A Digital thread establishes the link between the product and the DT and is expected to create a value stream of data across its lifecycle [16].

Computational Power: The improvement in computational power and processing speed are two of the main influential factors for Big Data processing and cloud computing. Powerful processors enable real-time updates required for the DT and reduce the time taken to solve the scenarios involving complex cases.

Machine learning and Artificial Intelligence (AI): The advancement in sensors and the increased amount of data generated resulted in rekindling of machine learning techniques and $\mathrm{AI}$ from the past decades to complement the current technologies [6]. The input domain knowledge required by the DT, to be in line with the system's performance, can be in the form of rules, ontologies, procedures, models or even sensor data [6], depending on the compatibility with the DT model. The output is generated by the DT model with the help of reasoning systems built around it and the advanced machine learning algorithms used. With the support of high processing speeds, machine learning algorithms can provide solutions in a shorter time, thus improving the efficiency of the entire problem-solving process.

Software: The progression in the data analytics software helps to convert the results produced by the DT into userfriendly visualizations, that encourage industry to opt for the DT approach for its products. Similarly, multiple platforms have made it easier for developing various models for DT, from designing the products to monitoring the manufacturing process and managing the maintenance activities.

The abovementioned technologies have made it possible for the broad application of DT in the industry, especially for the high-value products. The synergy among these technologies has helped increase efficiency, and save cost and time in every stage of a product's lifecycle. It is to be noted that these technologies also influence IVHM as described in Section I. This shows that the implementation of DT in IVHM would not require any drastic infrastructure change, rather a framework that provides state-of-the-art technologies that would benefit both the fields.

\section{ApPlications of A Digital Twin}

As stated before, the DT is a high-fidelity simulation model. It is capable of producing what-if scenarios for any given situation when provided with necessary data. In general, a DT serves as a virtual sensor that represents the product or the process at any instant and also as a feedback loop, providing data for further improvement. The DT is as powerful as the domain knowledge and specifications built into it, and as adaptive as the data it is supplied with. In general, the DT is adapted by the industries involved with high valued or highly customized products and processes due to the huge investment required to set up the infrastructure. With the help of enabling technologies mentioned in the previous section, industires have used DT for a variety of applications, adapted it to various scenarios. This section delivers some applications of DT developed in academia as well as by industry, based on the product's lifecycle stages, to present the versatile nature of the DT.

\section{A. Digital Twin in design \& development}

In the design and development phase of a product, the DT is generally used for testing, verification and validation $(V \& V)$ purposes, as well as to structural/ thermal and other analysis on the design. For example, Rios et al. [13] along with Airbus, used Dassault Systems V6 solutions to propose a framework for developing a DT model for an aircraft during the industrial design. The framework consisted of datasets generated from product-process-resources-systems, linked with each other, to provide an avatar of a dummy aircraft DA08. Data from 'asdefined', 'as-planned', 'as-prepared', 'as-built' and 'asmaintained' were linked along with the resources like 3D and simulation models and technical publications to develop the Digital Mock-Up of an aircraft assembly. Another example of the DT used in the design and development phase is its application with the help of CAE models by Siemens. In this case, the DT was used for various functions: i) predicting aerodynamic performance, blade deflection, and cooling flow rates of gas turbine blades, ii) predicting acoustic and structural loading, optimizing noise and vibration in epowertrain, and optimizing range and performance of electric vehicles, iii) predict fuel consumption of excavators for soil loading, and iv) enable V\&V of autonomous test driving [17]. Siemens has used the DT for Model-based system testing that connects the virtual testing model with bench testing and fieldtesting data to enable attribute specific evaluation throughout the development cycle. Siemens also uses the DT to support the testing process through anomaly detection process monitoring, and fault diagnosis [17].

\section{B. Digital Twin in Manufacturing}

The DT is used widely in manufacturing to monitor and optimize the production, to perform quality control on the products and predict defects in the assembly lines. Zheng et al. [18] developed a DT model of a welding production line with a 3D geometric, physical and kinematic model and connected it with the data from the production line to achieve interrelationship between the model and the real-time operation. This was used to monitor the welding production line to analyze the operation time and production cycle at each station and produce a real-time status warning. Similarly, Cai et al. [19] developed a DT of a vertical milling machine by data acquisition and information fusion techniques. They developed the DT with the characteristics of the machine using manufacturing data and sensory data from Hall-effect current and vibration sensors to monitor the operating 
conditions and to demonstrate the prediction capability for surface roughness model.

An industry-implemented DT use case is the 'Meggitt Modular Modifiable Manufacturing' or M4 [20] that explores the technologies of digital manufacturing, cloud computing, and IoT to address Meggitt's challenges of lack of flexibility and automation in the production lines that assemble aerospace products. The M4 framework combines the key components of the shop floor with sensors and connects them with data storage facilities, to carry out analysis required for the production line. The M4 framework was demonstrated by assembling the final stages of three critical safety-critical aerospace products, by carrying out what-if scenarios, optimizing the schedules, automating the part movements, and analyzing the factory performance at the end [20]. Likewise, Boeing uses Dassault Systems' 3D EXPERIENCE platform in its $777 \mathrm{X}$ composite wing center [21]. This new digital factory would help in streamlining the entire supply chain process by providing the real-time status of the factory, to optimize the plan for parts movement to match with the key performance indicators, to produce 'what-if' scenarios and compare the different methods for optimization, and to communicate with all the stakeholders about the updated plans for the movement. This will also help in loading and capacity simulation, decision making, and scheduling for parts production, and factory's performance management [21].

\section{Digital Twin in in-service monitoring}

The DT can be used to monitor the in-service performance of the product, that could give feedback to both maintenance as well as design stages. For example, Wang et al. [10] developed a DT for rotating machinery with a Finite Element Analysis model, along with a model updating strategy to accommodate for sensitivity between the physical and the digital model. They demonstrated fault diagnosis of the unbalanced condition of the rotor due to the varying operating conditions and environment. An industrial application of the DT in in-service monitoring is the Abrams tank track system. The DT was created by Endurica, along with TARDEC for the T-158LL back-pad of the Abrams tank track system and implemented via Abaqus solver and Endurica TL solver [22]. This DT was used to demonstrate the virtual evaluation of complex in-service conditions like self-healing, fatigue fault modes, thermal runaway failure mode, and the operating temperature; the results were found to be close to the experimental results of the back-pad component testing [22]. On the other hand, GE has built DT of the powerplants with physics-based and data-driven models to detect faults to improve the asset failure mode management and reduce unplanned downtime [15]. It also has built thermal DTs to assess the thermal efficiency and predict emission, as well as lifing DT to optimize the mission versus maintenance reliability of the power plant. GE uses its Predix platform to run the DTs. Predix is the key platform that ingests large scale data generated, carries out data processing, uses analytical models, and provides services via DTs at high execution speed [15]. Similarly, Sandvik Coromat has used Microsoft solutions like Azure IoT suite, Corona Intelligence and Dynamic 365 to develop a scalable DT in its production flow to connect to optimize the field service provided for the customers [23].
The examples described in this section show the versatile nature of the DT and that it is used by the high-value industries throughout a product's life cycle, in a variety of applications, ranging from digital mock-up to digital factories, and from predicting failure life to evaluating performance efficiency. The applications cited in this section are mainly from design and development, manufacturing, and in-service monitoring stages of the product life cycle. The following section discusses the applications of DT in maintenance, focusing exclusively on IVHM.

\section{DigITAL TWIN AND IVHM}

As mentioned earlier, IVHM is the paradigm shift to support CBM for vehicles. An IVHM system aims to assess the health of its host system, isolate faults, and predict useful life. The functioning of an IVHM system is influenced by the Open System Architecture - Condition Based Maintenance (OSA-CBM) framework. The OSA-CBM framework defines a series of steps (as shown in the middle layer of Fig. 3), that could be carried out to provide a maintenance solution which is adapted by the IVHM system [5]. Fig. 3 presents the basic process of an end-to-end IVHM system framework for an aircraft with the DT's role inscribed. The middle layer of Fig. 3 shows the steps followed by an IVHM system as defined by OSA-CBM. It starts with collecting data from the aircraft platform regarding a concerned subject which could either be a component (e.g., compressor blade), or a subsystem (e.g., gearbox assembly), or a system (e.g., engine). It then employs several data processing and manipulating techniques to extract information. This information is later used to assess health of the subject, detect the presence of any faults, diagnose and isolate faults, and predict the failure pattern and the subject's remaining useful life. The outcome from these three stages, i.e., condition monitoring, diagnosis, and prognosis, are sent for generating advisories which are then used by CBM to schedule maintenance programs for the concerned subject accordingly (bottom layer of Fig. 3).

To validate and ensure reliability of the results generated by the algorithms used for these three stages, the DT can be used as an alternate representation of the aircraft platform within the IVHM system (top layer of Fig. 3). To update the DT with the latest state of the aircraft's concerned subject, the data from the aircraft platform would undergo the same process of data acquisition and manipulation, similar to the initial steps followed by the IVHM system. This processed data, along with the context, could help in verification and optimization of the condition monitoring logic. Another advantage of inscribing DT to the IVHM system is that the DT would ideally possess knowledge of the entire system since its design stage (as discussed in Section II). One of the existing gaps in the field of IVHM is that the IVHM system is currently treated as an add-on to a matured design process of the asset [24]. The IVHM system is pointed to be more beneficial, if it is included at the earliest stage of the asset design process, strengthening the links of requirements and knowledge between the asset and the IVHM system. Hence, implementing DT, which represents the functioning of the physical asset, along with its contextual knowledge, would improve the overall performance of the IVHM system. One 
Figure 3: Digital Twin in an IVHM framework (adapted from [5])

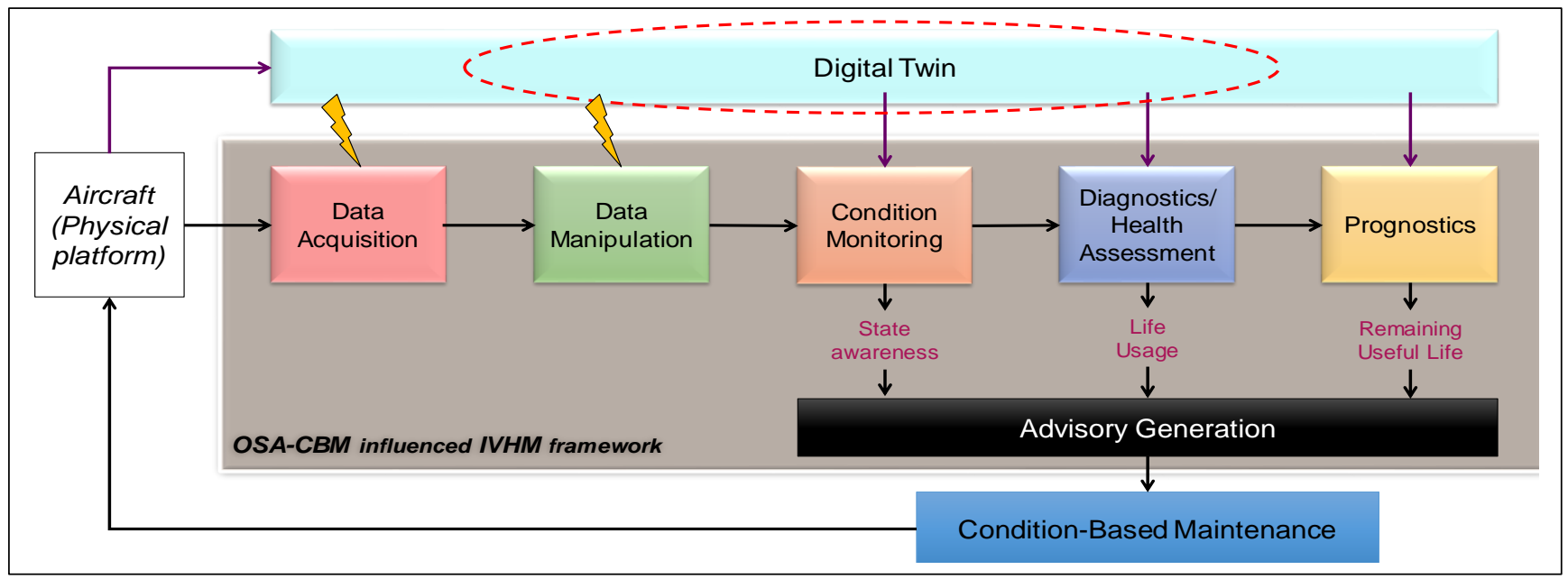

more advantage is the 'what-if' simulations that can be run in the DT at that point will augment the diagnosis and prognosis process. The better the results from the IVHM system, the better maintenance plans generated in CBM.

The DT, when first implemented by NASA, was planned to function with onboard IVHM systems to get health monitoring data to forecast the life of the systems and update maintenance schedules accordingly. Over the years, the role of DT evolved to supply chain streamlining, exterminating silos, and enabling visibility to stakeholders. However, the core of DT remains the same, i.e., to emulate the functioning of its physical counterpart, thus showcasing its health status. Even if a DT is used mostly in design and development or in the manufacturing stage of a product, it continually provides data in these stages that could be used for health monitoring eventually. Hence, the implementation of DT in the framework has become a natural step forward for the IVHM field. Some applications that use DT in IVHM are discussed in the rest of this section.

NASA and the United States (US) Airforce have developed several DTs for vehicle health monitoring. Tuegel [25] proposed an Airframe digital twin that would be a cradleto-grave model for designing and maintaining airframes. The Airframe digital twin helped in finding the probability of nonconformances during the design phase, which helped in keeping records of the individual aircraft through its dedicated computational model. This also helped in forecasting different scenarios of the airframe's performance and failure and predict the retirement schedule depending upon the individual aircraft's performance, rather than depending on the reliability statistics of the whole fleet. The uncertainty between the physical airframe and its DT was reduced by updating the service experience through Bayesian method. Another example is from Zakrajsek et al. [26], who developed a physics-based DT of an aircraft tire to monitor its health at the aircraft touchdown. In order to predict the tire wear during touchdown a physics-based equation involving slip wear rate was derived and combined with the empirical results to create a nonlinear touchdown response surface. The DT was used to predict the touchdown wear by varying the mean sink rate, yaw angle, and speed.
The F-35 Joint Strike Fighter developed by the US Airforce and Lockheed Martin has a state-of-the-art IVHM system that provides real-time health data during the flight to ground-based Autonomic Logistics Information System (ALIS), to enable the maintainers to be prepared for their activities when the aircraft lands [16]. ALIS then updates health data to all the local systems at Lockheed Martin. This, however, requires an enormous $\mathrm{V} \& \mathrm{~V}$ effort to ensure the integrity of data and also checks for hacking and other security-related issues, resulting in increased operations and maintenance costs. To save MRO cost, Lockheed Martin used ALIS to support operations and maintenance models and applied the Digital tapestry concept of data flow tightly woven to integrate the information from design and development into production (Digital thread), solving the early issues and leaning the production process like automated hole drilling. Lockheed Martin also applied this Digital tapestry concept to validate all the major component designs and enabled the conversion of SysML models to Simulink codes to reduce the gap between the systems and design perspectives [16].

Among the aerospace OEM industries, Rolls Royce uses a DT for engine health monitoring, to enable an IntelligentEngine, which is highly connected and contextually aware. Currently, the DT is used to carry out analysis under various possible instances of blade-off events in Trent engines [27]. Rolls Royce has also combined with Microsoft to enable predictive maintenance in Trent XWB turbofan jet engines, by employing data from historical feeds and real-time monitoring [23]. Similarly, GE has developed DT for aircraft landing gear by considering an instance as an initial model, followed by frequent updating of data [28]. The landing gear DT is made using asset data and time-series data. This DT is used to detect anomalies, forecast the next maintenance activities and predict remaining useful life of every subsystem of each landing gear, with the help of historical data collected and stored in the Predix platform and by performing predictive analytics [28].

Apart from aerospace, the DT has been used widely in automotive and maritime industries, as well as in railways. Tesla Motors creates a DT for every car it makes and uses it 
to provide fixes, software updates, and to schedule maintenance based on the car's usage and performance [29]. Likewise, Magargle et al. [30] from Ansys Inc. and Modelon Inc., developed a physics-based DT of automotive braking systems by combining $0-\mathrm{D}$ and $3-\mathrm{D}$ models with controls. They demonstrated the ability of this DT to differentiate the wear rates with normal and abnormal conditions while disconnecting the ABS controller, which would help in suggesting suitable maintenance schedules in advance for the braking system. They also used the DT to obtain sensor signatures for some fault conditions, which were further used to develop diagnostics for the automotive braking system.

The Danish Maritime Authority has adapted the DT for its maritime cluster, Blue Denmark, using 3D, simulation and analytical models, vessel documents and information models, operational data and sensor data, software-driven control algorithms and virtual communication networks [31]. The DTs are used to create value from individual elements like analysis for performance improvement, design verification and optimization, condition-based maintenance, decision support, safety assurance, testing and verification, and virtual operation. Currently, efforts are made to create a DT ecosystem that has the platform to integrate all these key elements to bring benefit to the stakeholders from the information contained in such an ecosystem [31]. GE has developed DT for railways, where, health index is calculated for network based on the defects found on the tracks and wayside [32]. Suitable maintenance plans are updated daily based on the health index. The DT is implemented for train also, for automatic control of throttle and dynamic brakes, optimization of speed and feedback on fuel consumption [32].

\section{Challenges, GaPS \& OpPORTUNities}

The applications presented in the previous sections show that the capabilities of DT have enabled it to contribute to highvalue industries in a number of sectors. The DT's ability to represent any product or process that could help provide feedback on health or performance or even verification, together with the maturity in the enabling technologies have the following potentials: i) save cost involved in design, testing, inspection, certification and unplanned maintenance, ii) improve the efficiency of knowledge transfer in a product lifecycle, iii) enable transparency in data handling, controlled sharing of data, and iv) to eliminate the silos across the different stakeholders and integrate and streamline the entire supply chain. On the other hand, the implementation of DT has a few challenges to be mitigated. This section discusses some of the challenges met by DT and its prospects in IVHM.

\section{A. Challenges faced by Digital Twin (DT) in IVHM}

One of the significant decision-making factors for implementing DT is the level of fidelity [9]. A dedicated DT, which is a replica of a physical system, consumes a large amount of time for development, requires more data and increases the cost involved. However, a low fidelity DT cannot reap the benefits of investment if it is not complex enough. A trade-off must be made to achieve the required level of fidelity in developing and managing the DT. Secondly, implementing DT requires suitable decisions and investments to be made regarding the enabling technologies. As described in the earlier sections, the technologies influencing DT and IVHM are very similar. The number of sensors used must be optimized to acquire all the required data as well as to not increase the overall weight of the vehicle. Data storage capacity must be factored in to accommodate Big Data, along with the infrastructure required for data management. High speed computations are required to power sophisticated machine learning algorithms; cloud computing facilities including the internet must be accounted for. With the infrastructure established, considering all these key technologies, implementation of the DT would become feasible within the IVHM framework. Another challenge faced by DT is security. In general, due to a large amount of data sharing on the cloud, the DT ecosystem is vulnerable to cyber-attack like hacking and phishing. The credibility of data generated has to be secured, and any possibilities of cyberattack should be diagnosed early on and prevented. Industries have started using technologies like Blockchain to secure data and prevent cyber-attacks [33]. One more issue that challenges the implementation of DT within IVHM is data sharing. Since health information about the systems of any asset is confidential to the suppliers, sharing such data without violating their intellectual property rights is not simple [34], which prevents the DT to be a complete representation of its physical counterpart. Further readings on the challenges faced by the DT implementation can be found in these works [[11], [25], [31]].

\section{B. Gaps \& Opportunities}

Even though introduced to the field of IVHM as early as 2012 , it is only in the last four years that the industry has taken DT into serious consideration, partly due to technology gaps in the industry. However, as the enabling technologies are becoming more affordable, there are opportunities for further research in this area. From the literature point of view, it is to be noticed that, the DTs developed are mostly at the components and subsystems level like fuel pump assembly and some DTs are developed at the system level like the fuel system. Only a very few works [[17], [35]] consider the integration of multiple DT models representing systems like the fuel system, the engine or the electrical power system to emulate their interactions at the level of an aircraft or any other vehicle. The intellectual properties of the suppliers could pose a challenge [34], as the lack of data would prevent the implementation of a fully representative DT. However, a 1-D black box representation that could connect to other such system level DT models for emulating an overall behavior of the vehicle could be pursued. When DT models representing various components and subsystems are integrated, the seamless simulation of any complex vehicle like an aircraft can be made possible. This will help in optimizing the results produced by the IVHM systems and in turn optimize the maintenance plans for these vehicles through CBM.

\section{CONCLUSION}

The DT has the potential to be the key to unlock many research areas related to IVHM. As one of the main objectives of IVHM is to integrate all available information to provide solutions to CBM, the DT can play a crucial role in being the platform that integrates all the information to project the health of the complex systems like an aircraft. A component 
or system level DT, integrated with other such DTs to emulate the functioning of a complex vehicle, combined with the technology that makes use of the data to assess health of its systems, thus becomes one of the latest enabling technologies of IVHM.

\section{REFERENCES}

[1] M. Grieves, "Origins of the Digital Twin Concept," 2016.

[2] E. Glaessgen and D. Stargel, "The Digital Twin Paradigm for Future NASA and U.S. Air Force Vehicles," in 53rd Structures, Structural Dynamics and Materials Conference, 2012, pp. 1-14.

[3] Gartner Inc, "Gartner Survey Reveals Digital Twins Are Entering Mainstream Use.” [Online]. Available:

https://www.gartner.com/en/newsroom/press-releases/2019-02-

20-gartner-survey-reveals-digital-twins-are-entering-mai. [Accessed: 28-Mar-2019].

[4] A. K. S. Jardine, D. Lin, and D. Banjevic, "A review on machinery diagnostics and prognostics implementing conditionbased maintenance," Mech. Syst. Signal Process., vol. 20, no. 7, pp. 1483-1510, 2006.

[5] L. Redding, "An Introduction to Integrated Vehicle Health Management - A Perspective from Literature," in Integrated Vehicle Health Management: Perspectives on an Emerging Field, I. K. Jennions, Ed. SAE International, 2011, pp. 17-26.

[6] C. M. Ezhilarasu, Z. Skaf, and I. K. Jennions, "The application of reasoning to aerospace Integrated Vehicle Health Management (IVHM): Challenges and opportunities," Prog. Aerosp. Sci., vol. 105, no. January, pp. 60-73, 2019.

[7] I. K. Jennions, "Introduction," in Integrated Vehicle Health Management: Perspectives on an Emerging Field, I. K. Jennions, Ed. SAE International, 2011, pp. 1-8.

[8] A. Madni, C. Madni, and S. Lucero, "Leveraging Digital Twin Technology in Model-Based Systems Engineering," Systems, vol. 7, no. 1, p. 7, 2019.

[9] F. Tao, M. Zhang, Y. Liu, and A. Y. C. Nee, "Digital Twin driven prognostics and health management for complex equipment," CIRP Ann. - Manuf. Technol., vol. 67, no. 1, pp. 169-172, 2018.

[10] J. Wang, L. Ye, R. X. Gao, C. Li, and L. Zhang, "Digital Twin for rotating machinery fault diagnosis in smart manufacturing," Int. J. Prod. Res., 2018.

[11] S. Singh and N. Higgins, "Challenges of Digital Twin in High Value Manufacturing," SAE Tech. Pap., vol. 01, no. 1928, pp. 110, 2018.

[12] Z. Liu, N. Meyendorf, and N. Mrad, "The role of data fusion in predictive maintenance using digital twin," in AIP Conference Proceedings 1949, 2018, vol. 020023, pp. 1-6.

[13] J. Rios, F. M. Morate, M. Oliva, and J. C. Hernandez, "Framework to support the aircraft digital counterpart concept with an industrial design view," Int. J. Agil. Syst. Manag., vol. 9, no. 3, 2016.

[14] Q. Qi and F. E. I. Tao, "Digital Twin and Big Data Towards Smart Manufacturing and Industry 4.0: 360 Degree Comparison," IEEE Access, vol. 6, pp. 3585-3593, 2018.

[15] General Electric, "GE Digital Twin," 2016. [Online]. Available: https://www.ge.com/digital/sites/default/files/Digital-Twin-forthe-digital-power-plant-.pdf. [Accessed: 04-Apr-2019].

[16] T. D. West and M. Blackburn, "Is Digital Thread / Digital Twin Affordable? A Systemic Assessment of the Cost of DoD's Latest Manhattan Project," Procedia Comput. Sci., vol. 114, pp. 47-56, 2017.

[17] H. Van der Auweraer, "Connecting physics based and data driven models : The best of two worlds," 2018. [Online]. Available: https://www.ima.umn.edu/materials/2017-2018/SW3.68.18/26842/IMA_2018_Van_der_Auweraer.pdf. [Accessed: 03Apr-2019].

[18] Y. Zheng, S. Yang, and H. Cheng, "An application framework of digital twin and its case study," J. Ambient Intell. Humaniz. Comput., vol. 10, no. 3, pp. 1141-1153, 2019.

[19] Y. Cai, B. Starly, P. Cohen, and Y. Lee, "Sensor data and information fusion to construct digital-twins virtual machine tools for cyber-physical manufacturing," Procedia Manuf., vol. 10, pp.
1031-1042, 2017.

[20] K. Jackson, K. Efthymiou, and J. Borton, "Digital manufacturing and flexible assembly technologies for reconfigurable aerospace production systems," Procedia CIRP, vol. 52, pp. 274-279, 2016.

[21] Dassault Systems, "Boeing \& Dassault Systemes Announce Extended Partnership.” [Online]. Available: https://www.cenit.com/fileadmin/dam/Events/PDFs/Aerospaceday2018/Partner_der_Luftfahrt_Wie_unterstuetzt_Dassault_Systeme s_die_digitale_Transformation_von_Airbus_und_Boeing_CENIT _Aerospace_Day.pdf. [Accessed: 05-Apr-2019].

[22] W. V Mars, M. Castanier, D. Ostberg, and W. Bradford, "Digital Twin for Tank Track Elastomers: Predicting Self-Heating and Durability," in Proceedings of the 2017 Ground Vehicle Systems Engineering and Technology Symposium (GVSETS), 2017.

[23] Microsoft Services, "The promise of a digital twin strategy," 2017. [Online]. Available: https://info.microsoft.com/rs/157GQE-382/images/Microsoft\%27s Digital Twin \%27How-To\%27 Whitepaper.pdf. [Accessed: 05-Apr-2019].

[24] I. K. Jennions, M. Esperon-Miguez, and O. Niculita, "Integrating IVHM and asset design," Int. J. Progn. Heal. Manag., vol. 7, no. 2, pp. 1-16, 2016.

[25] E. J. Tuegel, "The Airframe Digital Twin : Some Challenges to Realization," in 53rd AIAA/ASME/ASCE/AHS/ASC Structures, Structural Dynamics and Materials Conference, 2012, no. April, pp. $1-8$.

[26] A. J. Zakrajsek and S. Mall, "The Development and use of a Digital Twin Model for Tire Touchdown Health Monitoring," in 58th AIAA/ASCE/AHS/ASC Structures, Structural Dynamics, and Materials Conference, 2017, no. January, pp. 1-16.

[27] O. Pickup, "What is a digital twin and how does it keep RollsRoyce machines safe?" [Online]. Available: https://www.telegraph.co.uk/education/stemawards/digital/digital-twins-computer-modeling/. [Accessed: 02Apr-2019].

[28] GE Predix Case study, "Predictive Insights : Aircraft Landing Gear Prognostics." [Online]. Available: https://d154rjc49kgakj.cloudfront.net/Case Studies/Case Study_Landing Gear_FINAL.pdf. [Accessed: 01-Apr-2019].

[29] B. Schleich, N. Anwer, L. Mathieu, and S. Wartzack, "Shaping the digital twin for design and production engineering," CIRP Ann. - Manuf. Technol., vol. 66, no. 1, pp. 141-144, 2017.

[30] R. Magargle et al., "A Simulation-Based Digital Twin for ModelDriven Health Monitoring and Predictive Maintenance of an Automotive Braking System," in Proceedings of the 12th International Modelica Conference, 2017, pp. 35-46.

[31] Danish Maritime Authority, "Digital Twins for Blue Denmark," 2018. [Online]. Available: https://www.dma.dk/Documents/Publikationer/Digital Twin report for DMA.PDF. [Accessed: 04-Apr-2019].

[32] D. J. Rao, "Digital Twin for the Railway Network," GE Transportation- Digital Solutions. [Online]. Available: https://ondemand.gputechconf.com/gtc/2018/presentation/s8614-digitaltwin-for-the-railway-network.pdf. [Accessed: 04-Apr-2019].

[33] A. Chavali and C. Gottlieb, "Extending the Digital Treas with Blockchain in in Aerospace and Defense," 2018. [Online]. Available: https://www.accenture.com/_acnmedia/PDF75/Accenture-Extending-the-Digital-Thread-Blockchain-AD.pdf. [Accessed: 31-Mar-2019].

[34] "SAE JA6268: Design \& Run-Time Information Exchange for Health-Ready Components," SAE Int., Apr. 2018.

[35] C. M. Ezhilarasu, "An Integrated Reasoning Framework for Vehicle Level Diagnosis of Aircraft Subsystem Faults," in Annual Conference of Prognostics and Health Management Society, 2018. 
2019-11-28

Understanding the role of a Digital Twin in Integrated Vehicle Health Management (IVHM)

Ezhilarasu, Cordelia Mattuvarkuzhali

IEEE

Ezhilarasu CM, Skaf Z, Jennions IK. (2019) Understanding the role of a Digital Twin in Integrated Vehicle Health Management (IVHM). In: 2019 IEEE International Conference on Systems, Man, and Cybernetics (SMC), Bari, 6-9 October 2019

https://doi.org/10.1109/SMC.2019.8914244

Downloaded from Cranfield Library Services E-Repository 